\section{Eine Übersicht über Therapiefort- schritte in den letzten 50 Jahren beim Metastasierten Melanom}

\author{
D. Schadendorf ${ }^{1}$ \\ ${ }^{1}$ Klinik für Dermatologie, Universitätsklinikum Essen
}

Der schwarze Hautkrebs (malignes Melanom) ist ein von den Melanozyten abgeleiteter maligner Tumor. Trotz aller Anstrengungen zur Aufklärung und Prävention steigt die Zahl der Melanompatienten stetig. In Deutschland wird die Inzidenz aktuell auf ca. 20 Neuerkrankungen pro 100.000 Einwohner geschätzt; die Inzidenztrends zeigten einen dreifachen Anstieg über die letzten 30 Jahre. Auch wenn in Deutschland ein Großteil der Melanome in einem sehr frühen Stadium mit geringem Metastasierungsrisiko entdeckt wird, ist das Melanom auf Grund seiner Aggressivität im metastasierten Stadium für 80\% Todesfälle durch Hautkrebs verantwortlich. Das fehlende Ansprechen auf Chemotherapie hat das Dogma „hohe Therapieresistenz“ des metastasierten Melanoms begründet. Das 1975 zugelassene Alkylans, mit dem in klinischen Studien ein klinisches Ansprechen um 10\% und ein medianes Überleben von 8-11 Monaten reproduzierbar erreichbar wurde, war lange Zeit das einzige beim Melanom zugelassene Medikament. Die 2-Jahresüberlebensrate rangierte um $25 \%$.

In den letzten Jahren konnte aufgrund eines besseren biologischen Verständnisses des Melanoms sowie von Fortschritten sowohl in der zielgerichteten als auch in der Immuntherapie, verbunden mit der Zulassung verschiedener neuer Medikamente, die "Therapiefrustration" vertrieben und in eine neue Euphorie verkehrt werden. Beginnend in 2011 sind mehrere Therapeutika in Deutschland zugelassen worden, die sowohl ein verbessertes Tumoransprechen, als auch ein signifikant verlängertes Gesamtüberleben zeigen konnten.

\section{Stadieneinteilung und Diagnostik}

Melanozytäre Tumoren sollten, von wenigen Ausnahmen abgesehen, primär mit einem kleinen Sicherheitsabstand von 1-2 cm vollständig entfernt werden. Die Empfehlungen zur Ausbreitungsdiagnostik sind Abhängigkeit von der Tumoreindringtiefe, der Ulzeration und der Mitoserate. Eine lokoregionäre Metastasierung (Satelliten-, In-Transit- und lokoregionäre Lymphknotenmetastasen) wird als Stadium III klassifiziert. Die Lymphknotenmetastasierung tritt in etwa $20 \%$ der Melanompatienten in Deutschland auf und reduziert die 5-Jahresüberlebensrate auf 30-60\% in Abhängigkeit von der Anzahl der tumorbefallenen Lymphknoten sowie des nachgewiesenen Tumorvolumens. Hier werden effektive adjuvante Therapieverfahren neben dem Interferon gesucht.

\section{Therapieoptionen des fortgeschrittenen Melanoms}

Bis 2010 war für das metastasierte Melanom als systemische Therapie nur das Zytostatikum Dacarbazin in Deutschland zugelassen. Eine Überlebenszeitverlängerung war jedoch nie in einer randomisierten Studie gezeigt worden. Folglich war bei einer fortgeschrittenen Metastasierung (Stadium IV) die Prognose bis dahin sehr schlecht mit einer mittleren Überlebenszeit von 6-12 Monaten ab Zeitpunkt der Diagnose der Fernmetastasierung. Die zwischenzeitlich gewonnenen neueren Erkenntnisse auf molekularpathologischer als auch tumorimmunologischer Ebene haben zu der Entwicklung neuer Substanzen geführt ( $($ Tab. 1 ). Der gezielte Einsatz dieser Medikamente, abhängig vom Metastasierungsstatus des Patienten, der Dynamik der Metastasierung als auch vom Mutationsstatus des Patienten, wird die Melanomtherapie individualisieren und zu einer besseren Versorgungssituation aller Melanompatienten führen. Auch wenn die 2-, 3- und 5-Jahresüberlebensraten der entsprechend therapierten Melanompatienten steigen, ist es dennoch zu früh, um den vollen Umfang der Verbesserung abzuschätzen. Erst Langzeitdaten werden die Nachhaltigkeit der Therapieerfolge zeigen und darstellen, ob bzw. welche Patienten längerfristig von den neuen Therapieansätzen profitieren.

\section{Systemtherapien}

\section{Checkpoint-Blockade}

Zur erfolgreichen Entwicklung eines Tumors modulieren Melanomzellen auch das umgebende Mikromilieu inklusive des Immunsystems. Sie kooptieren beispielsweise Immuncheckpoint-Mechanismen, die normalerweise die Selbsttoleranz von Geweben regulieren. Durch Expression von PD-L1 und PD-L2, zwei Oberflächenliganden des programmed cell death 1 Proteins (PD-1), drosseln Melanomzellen die Aktivität von Effektor-T-Lymphozyten im Tumorgewebe. Da B-Lymphozyten und NK-Zellen ebenfalls PD-1 exprimieren, können auch sie durch diesen Mechanismus in ihrer Funktion gestört sein. Im Gegenzug ist das cytotoxic $\mathrm{T}$ lymphocyte-associated Antigen 4 (CTLA4) ausschließlich auf T-Zellen exprimiert, wodurch es die Amplitude der initialen T-Zell-Aktivierung nach Antigenpräsentation durch dendritische Zellen im Lymphknoten inhibiert.

Mit der therapeutischen Intervention der immunologischen Checkpoint-Kontrolle, d.h. der Interaktion zwischen Antigenpräsentation bzw. Tumorzellen und T-Lymphozyten ( $\triangle$ Abb. 1), ist ein Durchbruch in der onkologischen Therapie des Melanoms (wie auch bei anderen Tumorerkrankungen) gelungen. Ipilimumab (Yervoy ${ }^{\circledR}$ ), das gegen CTLA-4 gerichtet ist, ist seit 2011 in Deutschland auf dem Markt. In 2013 wurde die Zulassung um die Anwendung als Erstlinientherapie erweitert. Zwar sind die Ansprechraten mit deutlich unter 20\% (komplettes oder partielles Ansprechen) nicht viel besser als bei Dacarbazin. Jedoch war Ipilimumab die erste Substanz beim metastasierten Melanom überhaupt, für die ein signifikanter Überlebensvorteil gezeigt werden konnte, die bei etwa $20 \%$ der Patienten zu einer langfristigen Tumorkontrolle bis zu 10 Jahre führt, was den eigentlichen Vorteil dieser Therapie ausmacht. Ipilimumab führt durch die Bindung an den CTLA-4-Rezeptor zu einer erhöhten Aktivierung zytotoxischer T-Lymphozyten und damit zu einer gesteigerten Immunantwort, die sich gegen Tumorzellen, aber auch körpereigene Zellen richtet.

Nach Ipilimumab hat nun eine weitere Generation von Checkpoint-inhibitorischen Antikörpern, die PD1-Antikörper, für Aufsehen und Euphorie in der Onkologie gesorgt. Im Som- 
Tab.1 Auflistung der aktuell zugelassenen Therapeutika für das maligne Melanom und die Zulassung gemäß Fachinformation (Stand September 2016).

\begin{tabular}{|c|c|c|c|}
\hline & Handelsname & Substanzklasse & Zulassung \\
\hline \multirow[t]{2}{*}{ Interferon $\alpha$} & Intron $A^{\circledR}$ & Interferon $\alpha-2 b$ & $\begin{array}{l}\text { Als adjuvante Therapie beim malignen Melanom bei Patienten, die nach chirurg. } \\
\text { Eingriff tumorfrei, aber in hohem Maß rezidivgefährdet sind. }\end{array}$ \\
\hline & Roferon $^{\circledR}-A$ & Interferon $\alpha-2 a$ & $\begin{array}{l}\text { Malignes Melanom d. AJCC-Stadiums II (Breslow-Tumordicke }>1,5 \mathrm{~mm} \text {, ohne } \\
\text { Lymphknotenbeteiligung oder Hautausbreitung) bei Patienten, die nach einer } \\
\text { Tumorresektion krankheitsfrei sind. }\end{array}$ \\
\hline Dacarbazin & $\begin{array}{l}\text { Dacarbazin Lipomed }{ }^{\circledR} \\
\text { Detimedac }^{\circledR}\end{array}$ & Alkylans & Metastasiertes malignes Melanom. \\
\hline Vemurafenib & Zelboraf ${ }^{\circledR}$ & BRAF-Inhibitor & $\begin{array}{l}\text { Patienten mit BRAF-V600 Mutation-pos. nicht resezierbarem oder metastasier- } \\
\text { tem Melanom. }\end{array}$ \\
\hline Cobimetinib & Cotellic $^{\circledR}$ & MEK-Inhibitor & $\begin{array}{l}\text { In Kombination mit Vemurafenib zur Behandlung von erwachsenen Pat. mit } \\
\text { nicht-resezierbarem oder metastasiertem Melanom mit einer BRAF-V600- } \\
\text { Mutation. }\end{array}$ \\
\hline Dabrafenib & Tafinlar $^{\circledR}$ & BRAF-Inhibitor & $\begin{array}{l}\text { Patienten mit BRAF-V600 Mutation-pos. nicht resezierbarem oder metastasier- } \\
\text { tem Melanom. }\end{array}$ \\
\hline Trametinib & Mekinist ${ }^{\circledR}$ & MEK-Inhibitor & $\begin{array}{l}\text { Monotherapie oder in Kombination mit Dabrafenib zur Behandlung von er- } \\
\text { wachsenen Pat. mit nicht-resezierbarem oder metastasiertem Melanom mit } \\
\text { einer BRAF-V600-Mutation. }{ }^{\text {a }}\end{array}$ \\
\hline Ipilimumab & Yervoy $^{\circledR}$ & Anti-CTLA4-Antikörper & $\begin{array}{l}\text { Behandlung von fortgeschrittenen (nicht resezierbaren oder metastasierten) } \\
\text { Melanomen bei Erwachsenen. }\end{array}$ \\
\hline Nivolumab & Opdivo $^{\circledR}$ & Anti-PD-1-Antikörper & $\begin{array}{l}\text { Monotherapie oder in Kombination mit Ipilimumab des fortgeschrittenen, nicht- } \\
\text { resezierbaren oder metastasierten Melanoms bei Erwachsenen - auch in der } \\
\text { Erstlinientherapie. }\end{array}$ \\
\hline Pembrolizumab & Keytruda $^{\circledR}$ & Anti-PD-1-Antikörper & $\begin{array}{l}\text { Monotherapie des fortgeschrittenen, nicht-resezierbaren oder metastasierten } \\
\text { Melanoms bei Erwachsenen - auch in der Erstlinientherapie. }\end{array}$ \\
\hline
\end{tabular}

a Eine Trametinib-Monotherapie hat keine klinische Aktivität bei Patienten gezeigt, deren Erkrankung auf eine vorhergehende Therapie mit einem BRAF-Inhibitor nicht ansprach.

mer 2015 wurden sowohl Nivolumab (Opdivo ${ }^{\circledR}$ ) als auch Pembrolizumab (Keytruda ${ }^{\circledR}$ ) für das nicht-resezierbare oder metastasierte Melanom in Deutschland zugelassen. Die Zulassung von Nivolumab erstreckt sich inzwischen auch auf das nicht-kleinzellige Lungenkarzinom mit plattenepithelialer Histologie nach vorangegangener Chemotherapie. Mit Zulassungen bei anderen hämatologischen als auch onkologischen Tumoren ist kurzfristig zu rechnen.

Nivolumab und Pembrolizumab sind humane bzw. humanisierte Immunglobulin-G4-(IgG4) monoklonale Antikörper (HuMAb), die durch Bindung an den PD-1-Rezeptor die Interaktion des Rezeptors mit den Liganden PD-L1 und PD-L2 blockieren. Die Liganden PD-L1 und PD-L2 werden von Antigenpräsentierenden Zellen, aber auch von Tumoren oder anderen Zellen aus dem Mikromilieu des Tumors exprimiert. Durch Bindung der Liganden an den PD-1-Rezeptor, der ein negativer Regulator der T-Zellaktivität ist, kommt es zur Hemmung der T-Zellproliferation und Zytokinausschüttung. Bindet jedoch ein PD-1-Inhibitor an den PD-1-Rezeptor, so wird die negative Regulation aufgehoben; stattdessen kommt es zu einer Potenzierung der T-Zellreaktionen, einschließlich der Tumorabwehrreaktion ( $\bullet$ Abb. 1 ). Während Ipilimumab insbesondere in der Priming-Phase der T-Zellen bedeutsam ist, ist die Wirkung der PD-1-Inhibitoren auf die T-Zellen vor allem in der Effektorphase in der direkten Tumorumgebung zu sehen. Sowohl Ipilimumab als auch Nivolumab und Pembrolizumab werden als intravenöse Therapie gegeben.

Die Zulassung von Nivolumab erfolgte basierend auf den Ergebnissen von zwei Studien (http://www.ema.europa.eu/ema/ index.jsp?curl=pages/medicines/human/medicines/003985/ human_med_001876.jsp\&mid=WC0b01ac058001d124). In der CHECKMATE-066 Studie, einer randomisierten, doppelblinden Phase III-Studie, wurden 418 therapienaive Patienten mit BRAF-Wildtyp und metastasiertem Melanom eingeschlossen. Die Patienten erhielten entweder Nivolumab oder Dacarbazin. Das primäre Studienziel Gesamtüberleben wurde erreicht: Nivolumab-behandelte Patienten zeigten ein um 58\% reduziertes Risiko zu versterben und eine 1- bzw. 2-JahresÜberlebensrate von $73 \%$ bzw. $58 \%$ für Nivolumab und $42 \%$ bzw. 26\% für Dacarbazin bei einer Therapieabbruchrate von Nivolumab von $7 \%$. In einer weiteren randomisierten PhaseIII-Studie konnte zudem gezeigt werden, dass auch Patienten, die eine Vortherapie mit Ipilimumab erhalten haben, auf eine PD-1-Blockade mit Nivolumab ansprechen können (CHECKMATE-037). Trotz der Vortherapie mit Ipilimumab wurden nicht vermehrt Nebenwirkungen beobachtet, und die Rate an therapiebedingten Nebenwirkungen war vergleichbar mit den Ergebnissen der CHECKMATE-066-Studie.

Pembrolizumab wurde auf Grundlage der Ergebnisse von drei Studien zugelassen (http://www.ema.europa.eu/ema/index. jsp?curl=pages/medicines/human/medicines/003820/human _med_001886.jsp\&mid=WC0b01ac058001d124). In einer mehrarmigen, offenen Phase Ib-Studie (KEYNOTE-001) erhielten sowohl therapienaive als auch vorbehandelte Patienten Pembrolizumab. Die objektive Ansprechrate über alle Gruppen ( $n=655$ ) lag bei $38 \%$ mit einer Ansprechdauer von 1,9-10,8 Monaten. Die therapiebedingte Abbruchrate lag bei $3 \%$. In der dreiarmigen, randomisierten Phase II-Studie KEYNOTE-002 wurde Pembrolizumab mit einer Chemotherapie (Wahl des Prüfarztes) bei 540 Patienten mit Ipilimumab-vortherapierten Patienten verglichen (bei einer BRAF V600-Mutation war zusätzlich eine vorherige BRAF/MEK-Inhibitortherapie notwendig). Beide Pembrolizumab-Arme waren im PFS nach 6 bzw. 9 Monaten der Chemotherapie überlegen (34 bzw. 24\% Pembrolizumab im Vergleich zu 16 bzw. 8\% Chemotherapie). 


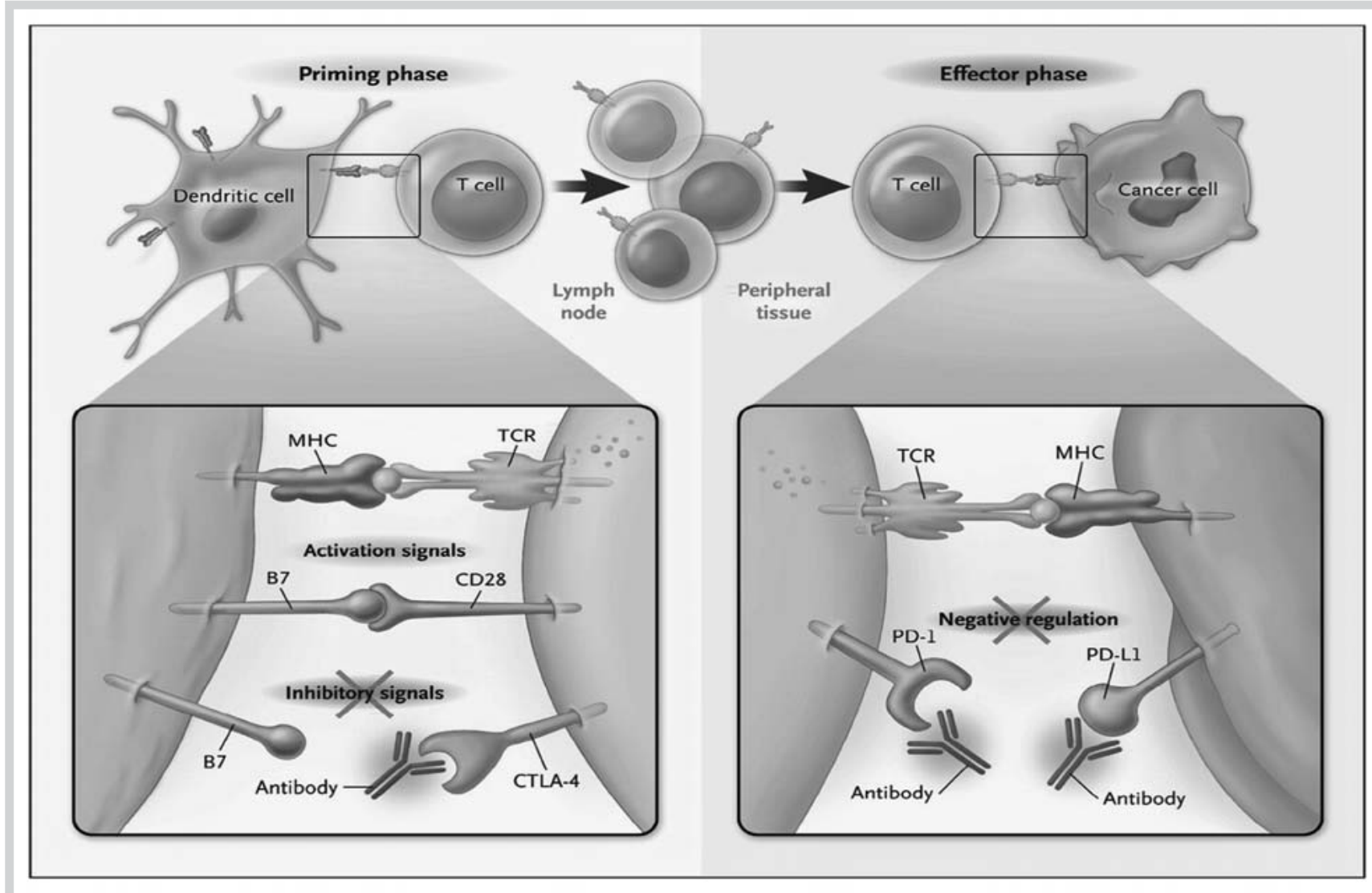

Abb. 1 Checkpoint-Inhibition beim Melanom (Abbildung aus Ribas A. N Engl J Med 2012;366:2517-2519.)

Auch in der KEYNOTE-006-Studie (randomisierte Phase IIIStudie) wurde Pembrolizumab untersucht und mit Ipilimumab verglichen. Bei der geplanten Zwischenanalyse zeigte Pembrolizumab einen signifikanten Vorteil sowohl für das PFS als auch das OS im Vergleich zu Ipilimumab. Das 1-Jahres-Gesamtüberleben von Pembrolizumab lag bei $74 \%$ im Vergleich zu 58\% bei Ipilimumab.

Im Gegensatz zu Ipilimumab konnten beide Anti-PD-1-Antikörper damit ein deutliches besseres Tumoransprechen (Ansprechraten um $40 \%$ ) zeigen und können selbst nach vorangegangenen Therapien (einschließlich Ipilimumab) klinisch effektiv sein bei vertretbarer Toxizität. Zwischenzeitlich wurde in einer Phase III-Studie (CHECKMATE-067) die synergistische Wirkung von Ipilimumab plus Nivolumab versus Monotherapie Ipilimumab bzw. Nivolumab untersucht. Die Kombinationstherapie zeigte ein deutlich höheres PFS (11,5 Monate) im Vergleich zur Monotherapie mit Ipilimumab (2,9 Monate) bzw. Nivolumab (6,9 Monate). Das Risiko, zu versterben bzw. einen Progress zu erleiden, war im Kombinationsarm im Vergleich zur Monotherapie mit Ipilimumab um 58\% reduziert. Die ORR lag bei 57,6\% für den Kombinationsarm (11,5\% Vollremission) vs. $19 \%$ im Ipilimumab-Arm (2,2\% Vollremission), und 43,7\% im Nivolumab-Arm (8,9\% Vollremission). Der BRAF-Status hatte keinen Einfluss auf die Ergebnisse. Letztlich muss aber abgewartet werden, ob sich diese Beobachtungen auch in den Gesamtüberlebensdaten, die für 2017 erwartet werden, zeigen wird. Dies ist insofern wichtig, als die Kombinationstherapie mit deutlich mehr und schwe- reren Nebenwirkungen einherging. Mehr als 50\% der Patienten, die die Kombinationstherapie erhalten hatten, entwickelten eine Grad 3/4 Toxizität, die häufig zur Hospitalisation führte. Interessanterweise scheinen diese Patienten, deren Therapie dann vorzeitig beendet werden musste, dennoch einen vergleichbaren klinischen Nutzen wie Patienten, die weiter behandelt wurden, zu zeigen.

Die erfolgversprechenden Ergebnisse dieser Studien werden ohne Zweifel dazu führen, dass sich tumorimmunologische Ansätze, insbesondere die Checkpoint-Blockade mit PD1-Antikörpern beim fortgeschrittenen Melanom, in den nächsten Jahren als wesentliche Therapiesäule etablieren werden.

\section{Blockade der Signalkaskade}

Das BRAF-Protein nimmt eine zentrale Rolle im MAPK-Signalweg ein ( $\boldsymbol{O} \mathbf{A} \mathbf{b} \mathbf{b} . \mathbf{2})$, der am normalen Wachstum und Überleben der Zellen beteiligt ist. Durch aktivierende Mutationen des BRAF-Gens kommt es zu einer Überaktivierung dieses Signalwegs, was zu exzessivem Zellwachstum und Krebs führen kann. Die routinemäßige Bestimmung von BRAF-Mutationen in Tumorgewebe ist essenziell, um für Patienten die geeignete zielgerichtete Therapien auszuwählen. Parallel zur Entschlüsselung der Bedeutung des MAPK-Signalwegs für Tumoren wurden selektive BRAF-Inhibitoren entwickelt, von denen zwei Substanzen, Vemurafenib (Zelboraf ${ }^{\oplus}$ ) und Dabrafenib (Tafinlar ${ }^{\circledR}$ ), aufgrund des Nachweises ihrer Überlegenheit (Ansprechrate, Tumorkontrolle, Gesamtüber- 


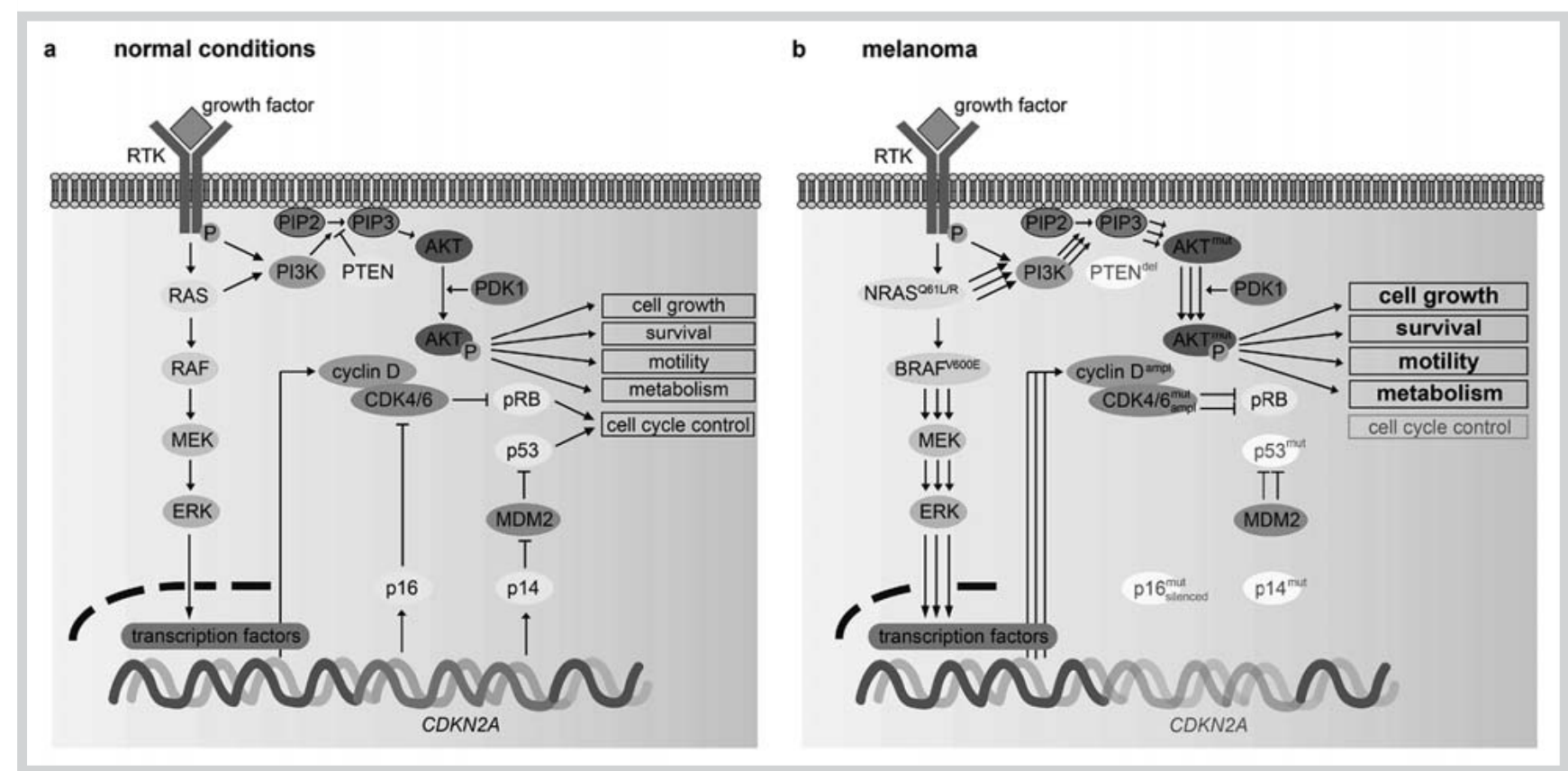

Abb. 2 Wichtige Signalwege beim Melanom

leben) gegenüber der traditionellen Chemotherapie mit Dacarbazin seit 2012 bzw. 2013 in Deutschland zugelassen sind. Ein Problem der BRAF-Inhibitoren ist die Resistenzentwicklung, die zu Überlegungen führte, den MAPK-Signalweg zusätzlich an weiteren Stellen zu inhibieren. Als weiterer Angriffspunkt wird beispielsweise das nachgelagerte MEK-Protein ( $\triangle \mathbf{A b b}$. 2) gesehen. Unterschiedliche MEK-Inhibitoren wurden und werden in klinischen Studien untersucht (u.a. Binimetinib, Cobimetinib und Trametinib).

Durch eine Kombination von BRAF- bzw. MEK-Inhibitoren gelingt eine duale Inhibition des MAPK-Signalwegs, die einerseits eine Resistenzentwicklung verzögert, andererseits eine Reduktion von BRAF-spezifischen Nebenwirkungen erreicht. In drei unabhängigen Phase III-Studien (Kombination von entweder Dabrafenib mit Trametinib oder Vemurafenib mit Cobimetinib) wurde sowohl eine weitere Verbesserung des klinischen Ansprechens, eine verbesserte Tumorkontrolle als auch eine Überlebensverbesserung gesehen. Das Gesamtüberleben liegt mit diesen Kombinationstherapien im Median deutlich über 2 Jahren und das mediane Überleben nach 3 Jahren bei $44 \%$ für die Studienpopulation.

Diese Ergebnisse mit Dabrafenib plus Trametinib haben inzwischen zur Zulassung (September 2015) von Trametinib in Kombination mit Dabrafenib geführt (http://www.ema.europa.eu/ema/index.jsp?curl=pages/medicines/human/medicines/002643/human_med_001772.jsp). Für Cobimetinib in Kombination mit Vemurafenib liegt seit Dezember 2015 eine Zulassung vor.
Interessenkonflikte: Prof. Dr. Dirk Schadendorf hat von Amgen, Array, Bristol-Myers Squibb, Boehringer Ingelheim, GlaxoSmithKline, Grünenthal, LeoPharma, Merck Sharp \& Dohme, Merck-Serono, Novartis, Pfizer, Roche und Sysmex Honorare für Berater- und Vortragstätigkeit und Reisekostenunterstützung erhalten. Er hat Forschungsförderung von Bristol-Myers Squibb, MSD und Plexxikon erhalten.

Bibliografie

DOI http://dx.doi.org/10.1055/s-0042-112397

Drug Res 2016; 66, Suppl. 1: S31-S34

(c) Georg Thieme Verlag KG Stuttgart · New York .

ISSN 2194-9379

Korrespondenzadresse Prof. Dr. Dirk Schadendorf Direktor der Klinik für Dermatologie und Direktor des Westdeutschen Tumorzentrums (WTZ)

Universitätsklinikum Essen Hufelandstraße 55 45147 Essen dirk.schadendorf@uk-essen.de 